\title{
PROOF OF A THEOREM OF LITTLEWOOD AND PALEY
}

\section{A. ZYGMUND}

1. Introduction. In recent years, important results in the theory of Fourier series were obtained by Littlewood and Paley [3]. ${ }^{1}$ They used complex methods, and their main tool was an auxiliary function, $g(\theta)$, which they themselves had introduced.

Let $\phi(z)$ be any function regular for $|z|<1$. The real-valued and non-negative function $g(\theta)=g(\theta ; \phi)$ is defined by the formula

$$
g(\theta)=\left\{\int_{0}^{1}(1-\rho)\left|\phi^{\prime}\left(\rho e^{i \theta}\right)\right|^{2} d \rho\right\}^{1 / 2}, \quad 0 \leqq \rho<1 .
$$

The integral on the right is finite or infinite, but always has meaning.

Let $f(\theta)$ be any $L$-integrable function of period $2 \pi$, and let $f(\rho, \theta)$ be the Poisson integral of $f$. Thus

$$
f(\rho, \theta)=\frac{1}{\pi} \int_{0}^{2 \pi} f(u) P(\rho, \theta-u) d u,
$$

where $P(\rho, t)=\left(1-\rho^{2}\right) / 2\left(1-2 \rho \cos t+\rho^{2}\right)$ is the Poisson kernel. If $\bar{f}(\rho, \theta)$ is the harmonic function conjugate to $f(\rho, \theta)$ and vanishing at the origin, and if we set

$$
\phi(z)=f(\rho, \theta)+i \bar{f}(\rho, \theta), \quad z=\rho e^{i \theta},
$$

the function (1.1) will sometimes be denoted by $g(\theta ; f)$.

The function $g(\theta)$ is suggested by some heuristic argument (see $[3, I])$. It does not seem to possess any obvious geometric significance, although it has a majorant, $s(\theta)$, with a simple geometric meaning. The reader interested in this problem is referred to papers $[4,7]$. In the present note we shall be exclusively concerned with the function $g(\theta)$.

As usual, by $H^{\lambda}$ we denote the class of functions $\phi(z)$ regular in $|z|<1$ and satisfying

$$
\int_{0}^{2 \pi}\left|\phi\left(\rho e^{i \theta}\right)\right|^{\lambda} d \theta=O(1), \quad 0 \leqq \rho<1 .
$$

As is well known, this condition implies almost everywhere the existence of the radial limit $\phi\left(e^{i \theta}\right)=\lim _{\rho \rightarrow 1} \phi\left(\rho e^{i \theta}\right)$.

Received by the editors January 8, 1945.

1 Numbers in brackets refer to the references cited at the end of the paper. 
Basic for the Littlewood-Paley theory (see also $[8]^{2}$ ) is the following result.

Theorem. Suppose that $\phi(z) \in H^{\lambda}, \lambda>0$, and that $g(\theta)=g(\theta ; \phi)$. Then

$$
\left\{\int_{0}^{2 \pi} g^{\lambda}(\theta) d \theta\right\}^{1 / \lambda} \leqq C_{\lambda}\left\{\int_{0}^{2 \pi}\left|\phi\left(e^{i \theta}\right)\right| \lambda d \theta\right\}^{1 / \lambda},
$$

with $C_{\lambda}$ depending on $\lambda$ only.

This result is proved in Littlewood and Paley [3, II] (where, unnecessarily, it is stated in a form valid for $\lambda>1$ only). The proof the authors give is far from simple. The inequality (1.3) is first established for $\lambda=2,4,6, \ldots$. In this case, the integral on the left of (1.3) is treated as a multiple ( $\lambda$-uple) integral, whose complexity obviously increases with $\lambda$. On the other hand, the passage from these special values of $\lambda$ to general $\lambda>0$ is simple.

The purpose of this note is to give another, perhaps slightly simpler proof of (1.3). The general idea of the proof will be as follows. (a) First we establish (1.3) for $\lambda=2$, which is immediate and quite familiar. (b) Next we show that the validity of (1.3) for any particular $\lambda$ implies its validity for any smaller and positive $\lambda$. (c) Finally, by a certain conjugacy argument we pass from the case $\lambda \leqq 2$ to $\lambda>2$.

The details of the proof are developed in the second section of this note. Part (b) of the proof is borrowed from Littlewood and Paley, and whatever novelty of the subsequent argument is restricted to part (c).

We shall also need the following two lemmas.

LEMmA 1. Let $\phi(z) \in H^{\lambda}, \lambda>0$, and let $\Phi(\theta)=\sup _{0 \leqq \rho<1}\left|\phi\left(\rho e^{i \theta}\right)\right|$. Then

$$
\left\{\int_{0}^{2 \pi} \Phi^{\lambda}(\theta) d \theta\right\}^{1 / \lambda} \leqq A_{\lambda}\left\{\int_{0}^{2 \pi}\left|\phi\left(e^{i \theta}\right)\right|^{\lambda} d \theta\right\}^{1 / \lambda} .
$$

LEMMA 2. For any regular function $\phi(z), z=\rho e^{i \theta}$,

$$
4\left|\phi^{\prime}(z)\right|^{2}=\Delta\left(|\phi|^{2}\right) \equiv \frac{1}{\rho} \frac{\partial}{\partial \rho}\left(\rho \frac{\partial}{\partial \rho}|\phi|^{2}\right)+\frac{1}{\rho^{2}} \frac{\partial^{2}}{\partial \theta^{2}}|\phi|^{2} .
$$

Lemma 1 is a very well known result of Hardy and Littlewood (see [1] or [6, p. 247]). Lemma 2 is also very well known. Its use in the sequel is analogous to that made by Littlewood and Paley in their proof of the theorem.

2 I take this opportunity to correct a misprint in $[8, \mathrm{II}]$. On p. 349 , line 2 , the denominator $(\log n)^{1 / \alpha}$ should be replaced by $(\log n)^{1 / \lambda}$. 
2. Proof of the theorem. It is enough to prove (1.3) for $\phi(z)$ regular in $|z| \leqq 1$. For if $0<R<1$, and if $g_{R}=g(\theta ; \phi(R z))$, then

$$
\begin{aligned}
g_{R}^{2}(\theta)=R^{2} \int_{0}^{1}(1-\rho)\left|\phi^{\prime}\left(R \rho e^{i \theta}\right)\right|^{2} d \rho & \leqq \int_{0}^{1}(1-\rho R)\left|\phi^{\prime}\left(\rho R e^{i \theta}\right)\right|^{2} d \rho \\
& =\int_{0}^{R}(1-\rho)\left|\phi^{\prime}\left(\rho e^{i \theta}\right)\right|^{2} d \rho .
\end{aligned}
$$

Thus $g_{R}(\theta) \leqq g(\theta), g_{R}(\theta) \rightarrow g(\theta)$, as $R \rightarrow 1$. If (1.3) is valid with $g, \phi(z)$ replaced by $g_{R}, \phi(R z)$, then on making $R$ tend to 1 we get (1.3) in full generality.

(a) To prove (1.3) for $\lambda=2$, let $\phi(z)=c_{0}+c_{1} z+c_{2} z^{2}+\cdots$ The series is absolutely and uniformly convergent for $|z| \leqq 1$, and

$$
\begin{aligned}
\frac{1}{2 \pi} \int_{0}^{2 \pi} g^{2}(\theta) d \theta & =\frac{1}{2 \pi} \int_{0}^{2 \pi} d \theta\left\{\int_{0}^{2 \pi}(1-\rho)\left|\phi^{\prime}\left(\rho e^{i \theta}\right)\right|^{2} d \rho\right\} \\
& =\int_{0}^{1}(1-\rho) d \rho \frac{1}{2 \pi} \int_{0}^{2 \pi}\left|\phi^{\prime}\left(\rho e^{i \theta}\right)\right|^{2} d \theta \\
& =\int_{0}^{1}(1-\rho) \sum_{\nu=0}^{\infty} \nu^{2}\left|c_{\nu}\right|^{2} \rho^{2 \nu-2} \phi d \rho \\
& =\sum_{\nu=1}^{\infty} \nu^{2}\left|c_{\nu}\right|^{2} \int_{0}^{1}(1-\rho) \rho^{2 \nu-2} d \rho=\sum_{\nu=1}^{\infty} \frac{\nu^{2}}{2 \nu(2 \nu-1)}\left|c_{\nu}\right|^{2} \\
& \leqq \frac{1}{2} \sum_{\nu=1}^{\infty}\left|c_{\nu}\right|^{2} \leqq \frac{1}{2} \cdot \frac{1}{2 \pi} \int_{0}^{2 \pi}\left|\phi\left(e^{i \theta}\right)\right|^{2} d \theta .
\end{aligned}
$$

(b) Suppose that (1.3) is established for some particular value of $\lambda$ and let $0<\kappa<\lambda$. Suppose that $\phi(z) \in H^{\kappa}$ and assume for a moment that $\phi(z)$ has no zeros for $|z|<1$. Let $\psi=\phi^{\kappa / \lambda}$. Thus $\phi^{\kappa}=\psi^{\lambda}$, and $\psi \in H^{\lambda}$. If we set $\Psi(\theta)=\sup _{0 \leqq p<1}\left|\psi\left(\rho e^{i \theta}\right)\right|, g(\theta)=g(\theta ; \phi), G(\theta)=g(\theta ; \psi)$, then

$$
\begin{aligned}
g^{2}(\theta)=(\lambda / \kappa)^{2} \int_{0}^{1}(1-\rho)|\psi|^{2(\lambda-\kappa) / \kappa}\left|\psi^{\prime}\right|^{2} d \rho=(\lambda / \kappa)^{2} \Psi^{2(\lambda-\kappa) / \kappa}(\theta) G^{2}(\theta) \\
\int_{0}^{2 \pi} g^{\kappa} d \theta \leqq(\lambda / \kappa)^{\kappa} \int_{0}^{2 \pi} \Psi^{\lambda-\kappa} G^{\kappa} d \theta \\
\leqq(\lambda / \kappa)^{\kappa}\left\{\int_{0}^{2 \pi} \Psi^{\lambda} d \theta\right\}^{(\lambda-\kappa) / \lambda}\left\{\int_{0}^{2 \pi} G^{\lambda} d \theta\right\}^{\kappa / \lambda}
\end{aligned}
$$

by the inequality of Hölder. Since we assumed the validity of (1.3) 
for our particular $\lambda$,

$$
\begin{aligned}
&\left\{\int_{0}^{2 \pi} g^{\kappa} d \theta\right\}^{1 / \kappa} \leqq(\lambda / \kappa)\left\{A_{\lambda}\left(\int_{0}^{2 \pi}\left|\psi\left(e^{i \theta}\right)\right|^{\lambda}\right)^{1 / \lambda}\right\}^{(\lambda-\kappa) / \kappa} \\
& \cdot C_{\lambda}\left\{\int_{0}^{2 \pi}\left|\psi\left(e^{i \theta}\right)\right|^{\lambda} d \theta\right\}^{1 / \lambda} \\
& \leqq(\lambda / \kappa) A_{\lambda}^{(\lambda-\kappa) / \kappa} C_{\lambda}\left\{\int_{0}^{2 \pi}\left|\psi\left(e^{i \theta}\right)\right|^{\lambda}\right\}^{1 / \kappa} \\
&=(\lambda / \kappa) A_{\lambda}^{(\lambda-\kappa) / \kappa} C_{\lambda}\left\{\int_{0}^{2 \pi}\left|\phi\left(e^{i \theta}\right)\right|^{\kappa} d \theta\right\}^{1 / \kappa} .
\end{aligned}
$$

This is (1.3), with $\kappa$ instead of $\lambda$, and with $C_{\kappa}=(\lambda / \kappa)^{1 / 2} A_{\lambda}^{(\lambda-\kappa) / \kappa} C_{\lambda}$.

Suppose now that $\phi(z)(\not \equiv 0)$ does have zeros in $|z|<1$. It is well known (see [2]) that then $\phi(z)=\phi_{1}(z)+\phi_{2}(z)(|z|<1)$, where $\phi_{1}$ and $\phi_{2}$ have no zeros, and $\left|\phi_{\kappa}(z)\right| \leqq 2|\phi(z)|$, for $k=1$, 2. If $g_{k}=g\left(\theta ; \phi_{k}\right)$, then $g \leqq g_{1}+g_{2}$, by the inequality of Minkowski. Thus

$$
\begin{aligned}
\int_{0}^{2 \pi} g^{\kappa} d \theta & \leqq 2^{k}\left\{\int_{0}^{2 \pi} g_{1}^{\kappa} d \theta+\int_{0}^{2 \pi} g_{2}^{k} d \theta\right\} \\
& \leqq 2^{\kappa}\left\{C_{\kappa}^{\kappa}\left(\int_{0}^{2 \pi}\left|\phi_{1}\right|^{\kappa} d \theta+\int_{0}^{2 \pi}\left|\phi_{2}\right|^{\kappa} d \theta\right)\right\} \\
& \leqq 2^{2 \kappa+1} C_{k}^{\kappa} \int_{0}^{2 \pi}\left|\phi\left(e^{i \theta}\right)\right|^{\kappa} d \theta
\end{aligned}
$$

This completes the proof of (b). In particular, (1.3) is established for $0<\lambda \leqq 2$.

(c) On account of (b), it is enough to prove (1.3) for $\lambda \geqq 4$. Let $\mu$ be defined by

$$
1 /(\lambda / 2)+1 / \mu=1 \text {. }
$$

Thus $1<\mu \leqq 2$, since $\lambda \geqq 4$. Let $\xi(\theta)$ denote any non-negative function such that $M_{\mu}[\xi]=\left(\int_{0}^{2 \pi} \xi^{\mu} d \theta\right)^{1 / \mu} \leqq 1$. Then

$$
M_{\lambda}^{2}[g]=\left\{\int_{0}^{2 \pi}\left(g^{2}\right)^{\lambda / 2} d \theta\right\}^{2 / \lambda}=\sup _{\xi} \int_{0}^{2 \pi} g^{2} \xi d \theta,
$$

where $g=g(\theta ; \phi)$. We may even restrict $\xi$ to trigonometric polynomials, without invalidating (2.1). Let us fix any such polynomial $\xi(\theta)$, and let us write $\gamma(\theta)=g(\theta ; \xi)$. If $\zeta(z)$ denotes the analytic function whose real part is the Poisson integral $\xi(\rho, \theta)$ of $\xi(\theta)$ and whose imaginary part vanishes at the origin, then $M_{\mu}\left[\zeta\left(e^{i \theta}\right)\right] \leqq R_{\mu} M_{\mu}[\xi] \leqq R_{\mu}$ by 
the very well known result of M. Riesz [5]. Here $R_{\mu}$ depends on $\mu$ only. Thus, observing that (1.3) has already been proved for $\lambda$ between 1 and 2 , we get

$$
M_{\mu}[\gamma] \leqq C M_{\mu}[\zeta] \leqq C R_{\mu} M_{\mu}[\xi] \leqq C R_{\mu},
$$

where $C=\sup _{1 \leqq \lambda \leqq 2} C_{\lambda}$ is an absolute constant.

We write

$$
\begin{aligned}
\int_{0}^{2 \pi} g^{2}(\theta) \xi(\theta) d \theta & =\int_{0}^{1}(1-\rho)\left\{\int_{0}^{2 \pi}\left|\phi^{\prime}\left(\rho e^{i \theta}\right)\right|^{2} \xi(\theta) d \theta\right\} d \rho \\
& =2 \int_{0}^{1}\left(1-\rho^{2}\right)\left\{\int_{0}^{2 \pi}\left|\phi^{\prime}\left(\rho^{2} e^{i \theta}\right)\right|{ }^{2} \xi(\theta) d \theta\right\} \rho d \rho \\
& \leqq 4 \int_{0}^{2 \pi}(1-\rho)\left\{\int_{0}^{2 \pi}\left|\phi^{\prime}\left(\rho^{2} e^{i \theta}\right)\right|{ }^{2} \xi(\theta) d \theta\right\} d \rho .
\end{aligned}
$$

The function $w(z)=\left|\phi^{\prime}(z)\right|^{2}$ being subharmonic,

$$
\begin{aligned}
w\left(\rho^{2} e^{i \theta}\right) & \leqq \frac{1}{\pi} \int_{0}^{2 \pi} w\left(\rho e^{i u}\right) P(\rho, \theta-u) d u, \\
\int_{0}^{2 \pi} w\left(\rho^{2} e^{i \theta}\right) \xi(\theta) d \theta & \leqq \frac{1}{\pi} \int_{0}^{2 \pi} \xi(\theta)\left\{\int_{0}^{2 \pi} w\left(\rho e^{i u}\right) P(\rho, \theta-u) d u\right\} d \theta \\
& =\int_{0}^{2 \pi} w\left(\rho e^{i u}\right) \xi(\rho, u) d u,
\end{aligned}
$$

$$
\int_{0}^{2 \pi} g^{2}(\theta) \xi(\theta) d \theta \leqq 4 \int_{0}^{1}(1-\rho)\left\{\int_{0}^{2 \pi}\left|\phi^{\prime}\left(\rho e^{i \theta}\right)\right| 2 \xi(\rho, \theta) d \theta\right\} d \rho .
$$

By Lemma 2, $4\left|\phi^{\prime}\right|^{2}=\Delta|\phi|^{2}$. The formula

$$
\Delta U=\frac{1}{\rho} \frac{\partial}{\partial \rho}\left(\rho \frac{\partial U}{\partial \rho}\right)+\frac{1}{\rho^{2}} \frac{\partial^{2} U}{\partial \theta^{2}}=\rho^{-1} U_{\rho}+U_{\rho \rho}+\rho^{-2} U_{\theta \theta}
$$

implies for any functions $a(\rho, \theta), b(\rho, \theta)$

$$
\Delta(a b)=a \Delta b+b \Delta a+2\left(a_{\rho} b_{\rho}+\rho^{-2} a_{\theta} b_{\theta}\right) .
$$

Thus, taking $a=|\phi|^{2}, b=\xi(\rho, \theta)$, and observing that $\Delta \xi=0$, we get

$$
4\left|\phi^{\prime}\right|^{2} \xi=\Delta\left(|\phi|^{2} \xi\right)-2\left\{|\phi|_{\rho}^{2} \xi_{\rho}+\rho^{-2}|\phi|_{\theta}^{2} \xi_{\theta}\right\} .
$$

It follows that the right-hand side of (2.3), which is non-negative, does not exceed 


$$
\begin{aligned}
& \int_{0}^{2 \pi} \int_{0}^{1}(1-\rho) \Delta\left(|\phi|^{2} \xi\right) \rho d \rho d \theta \\
& +4 \int_{0}^{2 \pi} \int_{0}^{1}(1-\rho)|\phi|\left(\left|\phi_{\rho}\right|\left|\xi_{\rho}\right|+\rho^{-2}\left|\phi_{\theta}\right|\left|\xi_{\theta}\right|\right) d \rho d \theta=A+B
\end{aligned}
$$

say. Since $|\phi| \leqq \Phi,\left|\phi_{\rho}\right|=\left|\phi^{\prime}\right|,\left|\rho^{-1} \phi_{\theta}\right|=\left|\phi^{\prime}\right|,\left|\xi_{\rho}\right| \leqq\left|\zeta^{\prime}\right|,\left|\rho^{-1} \xi_{\theta}\right|$ $\leqq\left|\zeta^{\prime}\right|$, we find

$$
\begin{aligned}
B & \leqq 8 \int_{0}^{2 \pi} \Phi(\theta) d \theta\left\{\int_{0}^{1}(1-\rho)\left|\phi^{\prime}\right|\left|\zeta^{\prime}\right| d \rho\right\} \\
& \leqq 8 \int_{0}^{2 \pi} \Phi(\theta) g(\theta) \gamma(\theta) d \theta \\
& \leqq 8 M_{\lambda}[\Phi] M_{\lambda}[g] M_{\mu}[\gamma] \leqq 8 C A_{\lambda} R_{\mu} M_{\lambda}[g] M_{\lambda}\left[\phi\left(e^{i \theta}\right)\right] .
\end{aligned}
$$

Here we use Schwarz's inequality, Hölder's inequality with the three indices $\lambda, \lambda, \mu$, and the inequality (2.2). To $A$ we apply the first formula (2.4) and note that, owing to periodicity, the integral of $\left(|\phi|^{2} \xi\right)_{\theta \theta}$ over $(0,2 \pi)$ is zero. Thus

$$
\begin{aligned}
A & =\int_{0}^{2 \pi} d \theta \int_{0}^{1}(1-\rho) \frac{\partial}{\partial \rho}\left(\rho \frac{\partial}{\partial \rho}|\phi|^{2 \xi}\right) d \rho \\
& =\int_{0}^{2 \pi} d \theta \int_{0}^{1} \rho \frac{\partial}{\partial \rho}\left(|\phi|^{2} \xi\right) d \rho \\
& =\int_{0}^{2 \pi}\left\{\left|\phi\left(e^{i \theta}\right)\right|^{2} \xi(\theta) d \theta-\int_{0}^{1}\left|\phi\left(\rho e^{i \theta}\right)\right|^{2} \xi(\rho, \theta) d \rho\right\} d \theta \\
& \leqq \int_{0}^{2 \pi}\left|\phi\left(e^{i \theta}\right)\right|^{2} \xi(\theta) d \theta \leqq M_{\lambda}^{2}\left[\phi\left(e^{i \theta}\right)\right] M_{\mu}[\xi] \leqq M_{\lambda}^{2}\left[\phi\left(e^{i \theta}\right)\right]
\end{aligned}
$$

From (2.1), (2.3), (2.5) and (2.6) follows

$$
M_{\lambda}^{2}[g] \leqq 8 C A_{\lambda} R_{\mu} M_{\lambda}[g] M_{\lambda}\left[\phi\left(e^{i \theta}\right)\right]+M_{\lambda}^{2}\left[\phi\left(e^{i \theta}\right)\right],
$$

so that $X=M_{\lambda}[g] / M_{\lambda}\left[\phi\left(e^{i \theta}\right)\right]$ does not exceed the largest root of the equation $X^{2}=8 C A_{\lambda} R_{\mu} X+1$. This proves (1.3) for $\lambda \geqq 4$, and so also for all $\lambda>0$.

3. Additional remark. The constant $A_{\lambda}$ in Lemma 1 is bounded for $\lambda \geqq \epsilon>0\left[1 ; 6\right.$, p. 247]. On the other hand, $R_{\mu}=O(\mu-1)^{-1}=O(\lambda)$ as $\mu \rightarrow 1$ (that is, $\lambda \rightarrow \infty$ ) $[5 ; 6$, p. 149]. Hence the largest root of the equation $X^{2}=8 C A_{\lambda} R_{\mu} X+1$ is $O(\lambda)$ for $\lambda \rightarrow \infty$. In other words, the constant $C_{\lambda}$ in (1.3) is bounded in every interval $0<\epsilon \leqq \lambda \leqq 1 / \epsilon$, and 
is $O(\lambda)$ for $\lambda \rightarrow \infty$. Thus $C_{\lambda} \leqq K \lambda$ for $\lambda \geqq 1$, where $K$ is an absolute constant.

Suppose that $\phi(z)$ is regular for $|z| \leqq 1$, and does not exceed 1 in absolute value there. Let $\alpha$ be any positive constant less than $1 / K e$. Summing the inequalities

$$
\frac{\alpha^{n}}{n !} \int_{0}^{2 \pi} g^{n} d \theta \leqq \frac{\alpha^{n}}{n !}(K n)^{n} \int_{0}^{2 \pi}\left|\phi\left(e^{i \theta}\right)\right|^{n} d \theta \leqq 2 \pi(\alpha K)^{n} n^{n} / n !
$$

for $n=0,1,2, \cdots$ we get

$$
\int_{0}^{2 \pi} \exp \{\alpha g(\theta)\} d \theta \leqq \beta .
$$

This inequality may be considered as the limiting case of (1.3) for $\lambda=\infty$. It may even be slightly strengthened. If $\phi(z)=u+i v$ is regular for $|z|<1$, and if $|u| \leqq 1$, then we have (3.1) where $\alpha$ and $\beta$ are positive absolute constants.

To show this, we observe that the proof of $\$ 2$ could be slightly modified, by using instead of Lemma 2 the relation

$$
2\left|\phi^{\prime}\right|^{2}=\Delta u^{2} \quad(\phi=u+i v)
$$

and instead of Lemma 1 the inequality

$$
\left\{\int_{0}^{2 \pi} U^{\lambda}(\theta) d \theta\right\}^{1 / \lambda} \leqq B_{\lambda}\left\{\int_{0}^{2 \pi}|u(1, \theta)|^{\lambda} d \theta\right\}^{1 / \lambda}, \quad \lambda>1,
$$

where $u(\rho, \theta)$ is any harmonic function satisfying $\int_{0}^{2 \pi}|u(\rho, \theta)|^{\lambda} d \theta$ $=O(1)$, and where $u(1, \theta)=\lim _{\rho \rightarrow 1} u(\rho, \theta), U(\theta)=\sup _{0 \leqq \rho<1} u(\rho, \theta)$. The coefficient $B_{\lambda}$ depends on $\lambda$ only and it is $O(1)$ as $\lambda \rightarrow \infty$. (Unlike $A_{\lambda}$, $B_{\lambda}$ tends to infinity as $\lambda \rightarrow 1$.) This modification of the argument of $\$ 2$ leads to the following analogue of (1.3)

$$
\begin{aligned}
\left\{\int_{0}^{2 \pi} g^{\lambda}(\theta) d \theta\right\}^{1 / \lambda} \leqq C_{\lambda}^{\prime}\left\{\int_{0}^{2 \pi}|u(1, \theta)| \lambda d \theta\right\}^{1 / \lambda} & (\lambda>1, \phi=u+i v)
\end{aligned}
$$

and here again $C_{\lambda}^{\prime}=O(\lambda)$ as $\lambda \rightarrow \infty$. Let $C_{\lambda}^{\prime} \leqq K \lambda$ for $\lambda \geqq 2$. If $|u| \leqq 1$, and if we consider (3.2) for $\lambda=2,4,6, \cdots$, we immediately obtain

$$
\int_{0}^{2 \pi} \exp \alpha g d \theta<2 \int_{0}^{2 \pi} \cosh \alpha g d \theta \leqq 4 \pi \sum_{0}^{\infty} \frac{\alpha^{2 n}}{(2 n) !}(K \cdot 2 n)^{2 n} \leqq \beta<\infty .
$$

This completes the proof. 


\section{REFERENCES}

1. G. H. Hardy and J. E. Littlewood, A maximal theorem with function-theoretic applications, Acta Math. vol. 54 (1930) pp. 81-116.

2. - Some new properties of Fourier constants, Math. Ann. vol. 97 (1926) pp. 159-209.

3. J. E. Littlewood and R.E.A. C. Paley, Theorems on Fourier series and power series. Part I, J. London Math. Soc. vol. 6 (1931) pp. 230-233; Part II, Proc. London Math. Soc. vol. 42 (1937) pp. 52-89; Part III, ibid. vol. 43 (1937) pp. 105-126.

4. J. Marcinkiewicz and A. Zygmund, On a theorem of Lusin, Duke Math. J. vol. 4 (1938) pp. 473-485.

5. M. Riesz, Sur les fonctions conjuguées, Math. Zeit. vol. 27 (1927) pp. 218-244.

6. A. Zygmund, Trigonometrical series, Warsaw, 1935. 204.

7. — On certain integrals, Trans. Amer. Math. Soc. vol. 55 (1944) pp. 170-

8. - On the convergence and summability of power series on the circle of convergence. I, Fund. Math. vol. 30 (1938) pp. 170-196; II, Proc. London Math. Soc. vol. 47 (1942) pp. 326-350.

MT. Holyoke College 